\title{
Specific inhibition of one DNMT1-including complex influences tumor initiation and progression
}

\author{
Mathilde Cheray ${ }^{1,2}$, Romain Pacaud ${ }^{1,2}$, Arulraj Nadaradjane ${ }^{1,2}$, François M Vallette ${ }^{1,2,3}$
} and Pierre-François Cartron ${ }^{1,2,4^{*}}$

\begin{abstract}
Background: Reactivation of silenced tumor suppressor genes by DNMT inhibitors has provided an alternative approach to cancer therapy. However, DNMT inhibitors have also been shown to induce or enhance tumorigenesis via DNA hypomethylation-induced oncogene activation and chromosomal instability. To develop more specific DNMT inhibitors for efficient cancer therapy, we compared the effects of peptides designed to specifically disrupt the interaction of DNMT1 with different proteins.

Findings: Our data indicated that the use of an unspecific DNMT inhibitor (5aza-2deoxycytidine), a DNMT1 inhibitor (procainamide) or peptides disrupting the DNMT1/PCNA, DNMT1/EZH2, DNMT1/HDAC1, DNMT1/DNMT3b and DNMT1/HP1 interactions promoted or enhanced in vivo tumorigenesis in a mouse glioma model. In contrast, a peptide disrupting the DNMT1/DMAP1 interaction, which per se did not affect tumor growth, sensitized cancer cells to chemotherapy/irradiation-induced cell death. Finally, our data indicated that the peptide disrupting the DNMT1/DMAP1 interaction increased the efficiency of temozolomide treatment.
\end{abstract}

Conclusion: Our data suggest that the DNMT1/DMAP1 interaction could be an effective anti-cancer target and opens a new avenue for the development of new strategies to design DNMT inhibitors.

Keywords: DNMT1, Epigenetic, DNA methylation, Glioma, GBM, Cell death

\section{Findings}

Numerous reports have identified the occurrence of global DNA hypomethylation as an oncogenic event in human tumorigenesis. Indeed, DNMT1 silencing and the disruption of the DNMT1/PCNA/UHRF1 complexes were described as two events that promote the initiation of tumorigenesis (malignant transformation from a non-tumor cell to tumor cell) via the induction of global DNA hypomethylation [1-4]. However, besides PCNA and UHRF1 proteins, DNMT1 has multiple partners of interaction, the loss of which could potentially promote the

\footnotetext{
* Correspondence: pierre-francois.cartron@univ-nantes.fr

'Centre de Recherche en Cancérologie Nantes-Angers, INSERM, U892, Equipe Apoptose et progression tumorale, Equipe labellisée Ligue Nationale Contre le Cancer, 8 quai moncousu, BP7021, 44007, Nantes, France

2Département de Recherche en Cancérologie, Université de Nantes, Faculté de Médecine, IFR26, F-4400, Nantes, France

Full list of author information is available at the end of the article
}

initiation of tumorigenesis or progression via the generation of the global DNA hypomethylation phenotype.

\section{Disruption of certain DNMT1/protein-x interactions}

Among the DNMT partners, we focused our analysis on the impact of tumorigenesis on the disruption of the DNMT1/PCNA, DNMT1/HDAC1, DNMT1/HP1 $\beta$, DN MT1/EZH2, DNMT1/Sp1, DNMT1/DMAP1 and DNM T1/DNMT3b complexes (Figure 1A) [5-14].

To investigate the impact of the disruption of these interactions on tumorigenesis, we transfected an astrocyte cell line (Astro\#40) and a glioma cell line (U87) with plasmid constructions encoding for peptides mimicking certain amino-acid regions implicated in the interactions existing between DNMT1 and DMAP1, DNMT3b, PCNA, EZH2, HDAC1, Sp1 and HP1 $\beta$ (Figure 1A and Additional file 1: Figure S1).



(c) 2013 Cheray et al.; licensee BioMed Central Ltd. This is an Open Access article distributed under the terms of the Creative Commons Attribution License (http://creativecommons.org/licenses/by/2.0), which permits unrestricted use, distribution, and reproduction in any medium, provided the original work is properly cited. 

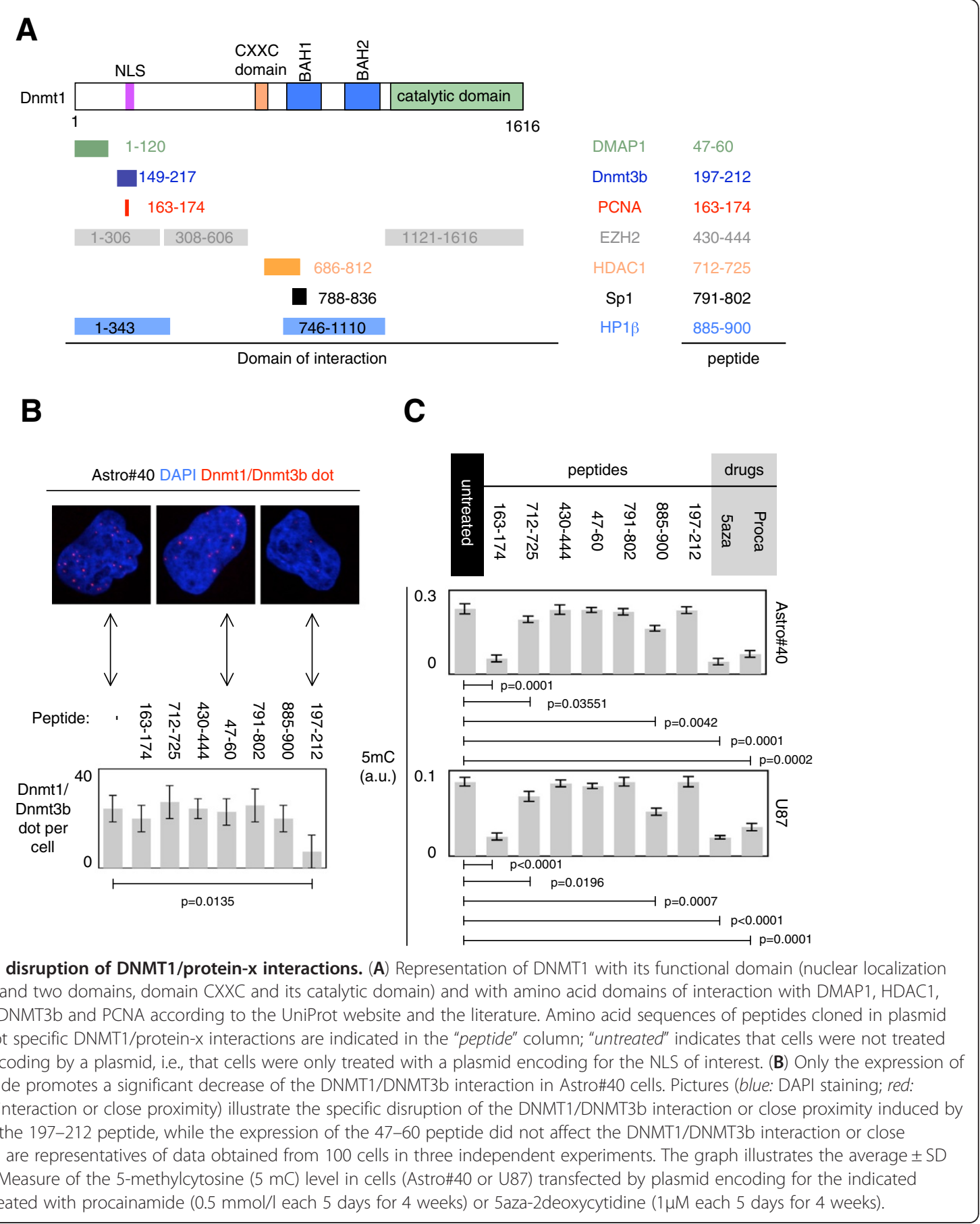

After 4 weeks of transfection/selection/amplification of cells, proximity ligation in situ assays (P-LISA) revealed that the 47-60, 197-212, 163-174, 430-444, 712-725, 791-802 and 885-900 peptides were specific to disrupting the interactions between DNMT1 and DMAP1, DNMT3b, PCNA, EZH2, HDAC1, Sp1 and HP1 $\beta$, respectively. For example, we observed in Astro\#40 that the 197-212 peptide disrupted the DNMT1/DNMT3B interaction, but not the DNMT1/HP1 $\beta$, DNMT1/HDAC1, DNMT1/ Sp1, DNMT1/EZH2 and DNMT1/DMAP1 interactions (Figure 1B). More generally, we noted that peptides designed to specifically disrupt an interaction disrupted only the targeted and expected interactions in Astro\#40 and U87 cells (Additional file 2: Figure S2). 
Impact of the disruption of DNMT1/protein-x interactions on the global DNA methylation level

We then investigated the impact of these disruptions on the global level of DNA methylation, i.e., on the 5-methylcytosine level. Two DNMT inhibitors (5aza-2deoxycytidine and procainamide) were used as these drugs induced global DNA hypomethylation (Figure 1C). In agreement with our previous reports, the 163-174 plasmid was used as a peptide that induced global DNA hypomethylation [3,4] (Figure 1C). Thus, we noted that the disruption of DNMT1/HDAC1 and DNMT1/HP1 $\beta$ interactions promoted a global decrease in the 5-methylcytosine level in Astro\#40 and U87 cells, while the disruption of the DNMT1/EZH2, DNMT1/Sp1 and DNMT1/DMAP1 interactions did not affect the 5-methylcytosine level in these cells (Figure 1C).

\section{Impact on tumorigenesis of the disruption of DNMT1/ protein-x interactions}

Tumorigenic assays performed in Swiss nude mice revealed that the subcutaneous injection of Astro\#40 cells treated 4 weeks with 5aza-2deoxycytidine and procainamide or transfected with plasmid encoding for the 163-174, 712-725 and 885-900 peptides generated tumor formation in $3 / 5,2 / 5,5 / 5,2 / 5$ and $2 / 5$ cases, respectively (Figure 2A). Thus, we observed that gliomagenesis is induced by the loss of DNMT1/PCNA, DNMT1/HDAC1 and DNMT1/HP1 $\beta$ interactions, i.e., under conditions that also induced a decrease in the global DNA methylation level. In parallel, we noted that the subcutaneous injections of U87 cells treated with 5aza-2deoxycytidine and procainamide or transfected with plasmid encoding for the 163-174, 712-725, 430-444, 885-900 and 197-212 peptides generated the formation of larger tumors as compared to untreated U87 cells (Figure 2B). These data suggest that the disruption of DNMT1/PCNA, DNMT1/ EZH2, DNMT1/DNMT3B, DNMT1/HDAC1 and DNM $\mathrm{T} 1 / \mathrm{HP} 1 \beta$ interactions acts such as an enhancer of tumorigenesis. Thus, our work stratifies the specific disruption of certain DNMT1/protein- $x$ interactions into two categories (Figure 2C). The first category includes the specific disruption of certain DNMT1/protein-x interactions acting as inducers and/or enhancers of gliomagenesis (DNMT1/ PCNA, DNMT1/HDAC1 and DNMT1/HP1ß, DNMT1/ EZH2, DNMT1/DNMT3b), i.e., acting as pro-tumori genic-specific disruption of DNMT1/protein-x interactions. The second category includes the specific disruption of DNMT1/protein-x interactions devoid of any action on gliomagenesis. This category is called neutral-tumori genic-specific disruption of DNMT1/protein-x interactions. Although absent in our data, we added a third category called anti-tumorigenic-specific disruption of DNMT1/protein-x interactions including the specific disruption of DNMT1/protein-x interactions promoting the decrease in tumorigenesis (Figure 2C).

\section{Focus on two disruptions of DNMT1/protein-x interactions devoid of action on gliomagenesis}

In the absence of any observations of anti-tumorigenicspecific disruptions of DNMT1/protein-x interactions, we next analyzed whether the specific disruption of a DN MT1/protein-x interaction devoid of action on gliomagenesis could have an impact on the response to the standard GBM treatment combining temozolomide (TMZ) and irradiation. For this, we measured the percentage of cell death induced by a TMZ + irradiation treatment of U87 cells transfected with the plasmids encoding for the 47-60 and 791-802 peptides (Additional file 3: Figure S3). We noted that the 47-60 peptide, which induced the disruption of the DNMT1/DMAP1 interaction, increased the percentage of $\mathrm{TMZ}+$ irradiation-induced cell death, while the 791-802 peptide, which induced the disruption of the DNMT1/Sp1 interaction, had no effect on the percentage of $\mathrm{TMZ}+$ irradiation-induced cell death (Figure 2D). In addition, our data also indicated that the 5aza-2deoxycytidine and procainamide treatments and the 163-174 peptide induced a phenotype resistant to $\mathrm{TMZ}+$ irradiation cell death since the percentage of TMZ-irradiation-induced cell death significantly decreased under these conditions (Figure 2D). Taken together, these data indicate that, among the considered peptides and DNMT1 inhibitors, only the specific 47-60-induced disruption of the DNMT1/DMAP1 interaction increased the percentage of $\mathrm{TMZ}+$ irradiation-induced cell death without promoting or increasing the tumorigenesis. Thus, we conclude that the specific inhibition of the DNMT1/DMAP1 interaction is a neutral-tumorigenic-specific inhibition of DNMT1/ protein- $x$ interaction harboring the capacity to sensitize cells to TMZ + irradiation-induced cell death.

\section{Disruption of DNMT1/DMAP1 interactions enhances the anti-tumor effect of TMZ treatment in mice}

To investigate this point, established tumors were treated with TMZ, TMZ $+47-60^{\text {DNMT1 }}$ and mutated $47-60^{\text {DNMT1 }}$ $\left(\mathrm{m} 47-60^{\mathrm{DNMT1}}\right)$ (Additional file 4 : Figure S4). Mice received 6 weeks of treatment as in treatment of human GBM, and tumor weight was analyzed 2 weeks after the end of the treatment. As illustrated in Figure 2E, we first noted that TMZ treatment reduced the tumor weigh of U87-induced glioma. Second, we observed that the addition of $47-60^{\text {DNMT1 }}$ increased the efficiency of TMZ treatment and decreased tumor growth, while m47-

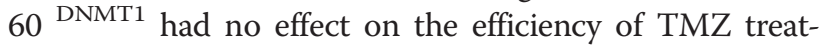
ment (since this peptide does not disrupt the DNMT1/ DNMAP1 interaction; Additional file 5: Figure S5). Thus, 
A



C

Impact of Dnmt1 inhibition or disruption of specific Dnmt1/protein-x interaction on tumorogenesis
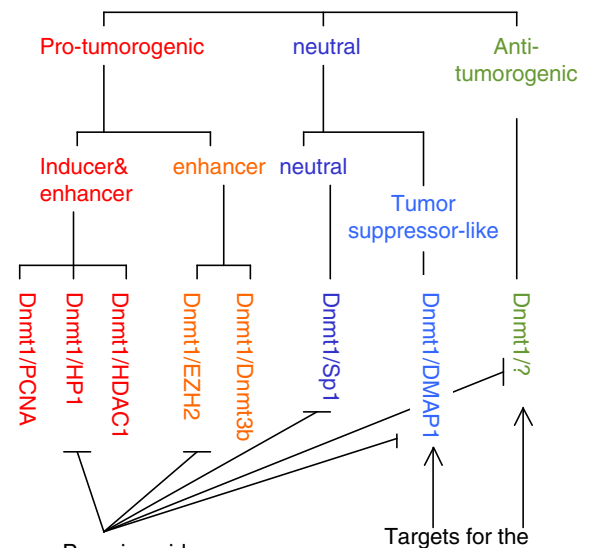

Procainamide (Dnmt1 inhibitor)

5aza-2deoxycytidine (unspecfifc Dnmt1 inhibitor)
B
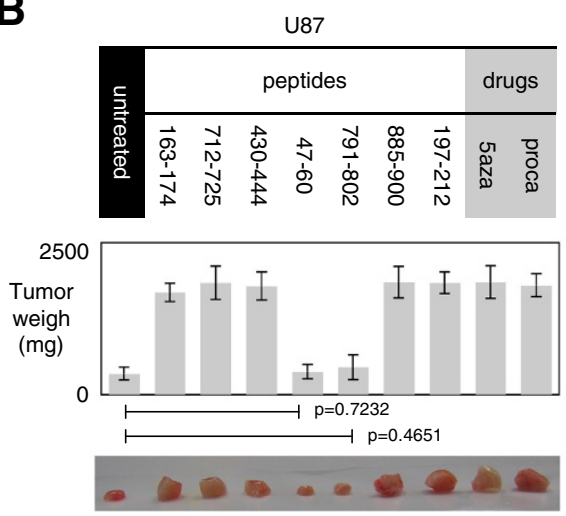

D
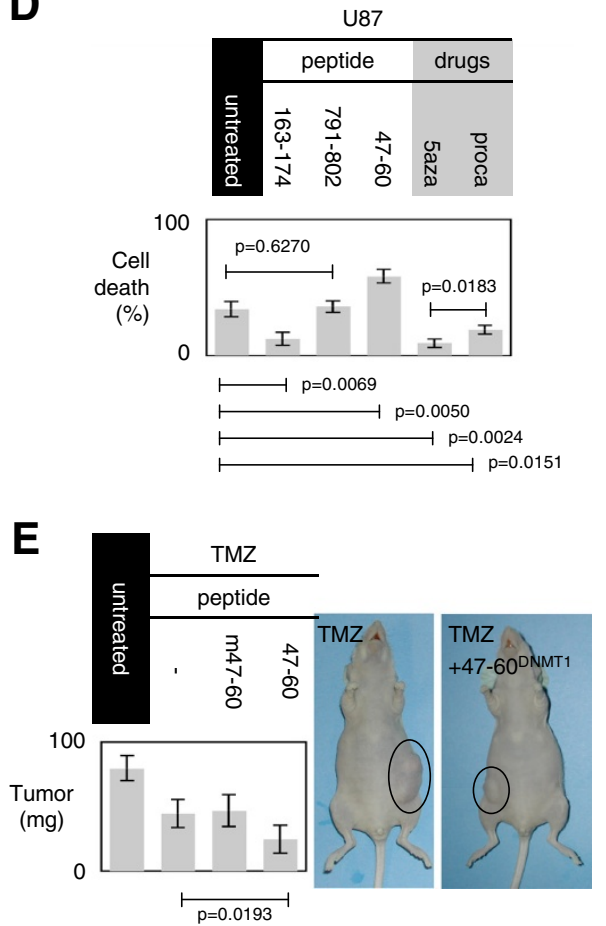

Figure 2 Impact of specific inhibition of DNMT1/protein-x interaction. (A) Tumorigenicity test of Astro\#40 cells transfected with plasmids encoding for indicated peptides or pre-treated with procainamide. For each condition, five subcutaneous injections of $10^{6}$ cells were performed in Swiss nude nice. Picture illustrates the tumor obtained (or not) 5 weeks after injections. (B) Tumorigenicity test of U87 cells transfected with plasmids encoding for indicated peptides or pre-treated with epigenetic drugs. For each condition, three subcutaneous injections of $10^{6}$ cells were performed in Swiss nude mice. Graph illustrates the average \pm SD of these data. Picture illustrates representative tumors obtained 4 weeks after injections. (C) Effect of different DNMT inhibitor strategies. DNA methylation governs the expression of tumor suppressor genes (TSG) but also of oncogenes and DNA repeat element and retrotransposons (whose demethylation and/or expression promotes the tumorigenesis). By using an unspecific DNMT inhibitors (5aza-2deoxycytidine) or a specific DNMT1 inhibitor (procainamide), we postulate that we induced the hypomethylation not only of TSG but also of the oncogenes and retrotransposons. By reducing the effect of DNMT1 inhibition to certain specific DNMT/protein-x interactions, we still keep the opportunity to promote the expression of the TSG without promoting the hypomethylationinduced activation of retrotransposons and oncogenes. (D) Analysis of TMZ + irradiation-induced cell death in U87 cells. Percentages of cell death were evaluated by using a trypan blue stain $0.4 \%$, and the Countess ${ }^{\circledast}$ Automated Cell Counter (Life Technology, France). Graph illustrates the average \pm SD of three independent experiments. (E) Impact of the addition of 47-60 DNMT1 on TMZ treatment in an in vivo model of glioma. After the tumor establishment, mice were treated with $\mathrm{TMZ}$ and $\mathrm{TMZ}+47-60^{\mathrm{DNMT1}}$ (Additional file 4: Figure S4). Two negative controls were used: "untreated" represents a treatment with DMSO "m47-60 DNMT1" symbolized a co-treatment using TMZ and mutated 47-60 DNMT1. Graph illustrates the average \pm SD of five independent experiments. 
our data identify $47-60^{\text {DNMT1 }}$ as an enhancer of TMZ treatment.

\section{Discussion}

To summarize, our data indicate that the specific inhibition of certain DNMT1/protein-x interactions (DNM T1/PCNA interaction, for example) and the use of a specific DNMT1 inhibitor (procainamide) or unspecific DN MT1 inhibitor (5-aza-2deoxycytidine) could be used as a treatment, acting as inducers and/or enhancers of tumorigenesis. Our data indicate that the specific inhibition of the DNMT1/DMAP1 interaction acts as a tumor suppressorlike event since the disruption of the DNMT1/DMAP1 interaction increased TMZ + irradiation-induced cell death without promoting the initiation and progression of tumorigenesis. Consequently, we distinguish between among the neutral-tumorigenic-specific inhibition of DNMT1/ protein-x interaction and the tumor suppressor-like neutral-tumorigenic-specific inhibition of DNMT1/ protein-x interaction (Figure $2 \mathrm{C}$ ).

Using this example, our data underline the necessity to consider the interaction partners of DNMT1 and not only the DNMT1 structure or activity to develop a DNMT1 inhibitor. In other terms, our data introduce, for the first time, the notion of protein/protein inhibition into the development of DNMT inhibitors. Indeed, without being innovative in the development of drugs or small molecules for a therapeutic application, this strategy is novel in the conception/research of DNMT inhibitors since the identification of DNMT inhibitors is, to date, based on docking-based virtual screening methods, the screening of natural products, the design and generation of derivatives of DNMT inhibitors already known, or molecular modeling of DNMT inhibitors by using crystal structure studies of DNMTs [15-19]. Thus, like ABT-737 and MI-129, two compounds targeting specific protein-protein interactions (pro-apoptotic/anti-apoptotic and p53/MDM2 interactions, respectively) have opened a new area in targeted therapy; our data argue that the targeting of certain DNMT1/protein-x interactions opens a new area in the development of targeted epigenetic therapy.

However, despite its promising character, the development of specific inhibitors of DNMT1/protein-x interactions requires the identification of tumor suppressor-like neutral-tumorigenic-specific inhibition of DNMT1/protein-x interaction or anti-tumorigenic-specific disruption of certain DNMT1/protein-x interactions. Thus, studies are ongoing in our laboratory to identify a DNMT1/protein- $\mathrm{x}$-including complex promoting the methylationinduced silencing of the tumor suppressor gene without being implicated in the methylation-induced silencing of oncogenes.
Concerning the use of 5aza-2deoxycytidine and procainamide, we are aware that the tumorigenesis processes associated with the use of these drugs are obtained after a long exposure. However, 4 weeks is not a long period on the scale of the majority of the chemotherapeutic treatments that last several months. Regardless, our data provide "a warning" concerning the use of DNA demethylating agents as an anti-cancer therapy and provide proof reinforcing the necessity of using specific DNMT inhibitors or unspecific DNMT1 inhibitors with an adequate and optimized dose schedule, as already described by several publications [20,21]. Besides, several publications also argue that the use of DNMT inhibitors could promote oncogene activation [22,23]. Thus, Chik and Szyf [22] report that 5aza-2deoxycytidine activated both silenced tumor suppressor genes and pro-metastatic genes by demethylation, raising the concern that it could promote metastasis [22]. Associated with our results, these data support the idea of developing specific DNMT1, particularly developing a specific inhibitor of DNMT1/protein-x interaction.

\section{Methods}

\section{Proximity ligation in situ assay (P-LISA)}

P-LISA is a technology permitting the visualization of stable and transient interactions at endogenous protein levels directly in situ [20]. Briefly, two primary antibodies raised in different species recognize the target antigen or antigens of interest. Species-specific secondary antibodies, called PLA probes, each with a unique short DNA strand attached to it, bind to the primary antibodies. When the PLA probes are in close proximity $(<40 \mathrm{~nm})$, the DNA strands can interact through a subsequent addition of two other circle-forming DNA oligonucleotides. After ligating the two added oligonucleotides, creating a circle DNA molecule, they are amplified via rolling circle amplification. After amplification, several-hundredfold replication of the DNA circle has occurred, and labeled complementary oligonucleotide probes highlight the product. The resulting high concentration of fluorescence in each single-molecule amplification product is easily visible as a distinct bright dot when viewed with a fluorescence microscope.

Cells were cultured for $24 \mathrm{~h}$ on cover slips. Cells were then fixed with $4 \%$ paraformaldehyde in PBS, $\mathrm{pH} 7.4$, for $15 \mathrm{~min}$ at room temperature. Permeabilization was performed with PBS containing 0.5\% Triton X-100 for 20 min at room temperature. Blocking, staining, hybridization, ligation, amplification and detection steps were realized according to the manufacturer's instructions (Olink Bioscience). All incubations were performed in a humidity chamber. Amplification and detection steps were performed in a dark room. Fluorescence was visualized by using the Axiovert 200M microscopy system (Zeiss, 
Le Pecq, France) with the ApoTome module (X63 and numerical aperture 1.4). Preparations were mounted using ProLong ${ }^{\circ}$ Gold antifade reagent with DAPI (InVitrogen, France). Picture acquisition was realized in structured illumination microscopy [24]. Finally, the images were analyzed using the freeware "BlobFinder" available for download from www.cb.uu.se/ amin/BlobFinder. Thus, we obtained the number of signals per nuclei since nuclei can be automatically identified.

\section{Plasmid construction and transfection}

To express peptides in cells, we subcloned in pcDNA3.3 (Life Technology, France) the sequences encoding for the indicated peptides. In addition, the NLS sequence (PKKKRKV) was added to the sequences encoding for the indicated peptides in order to address the peptides in the nucleus. Tranfections were next realized by using $2.10^{5}$ cells, $5 \mu \mathrm{g}$ of plasmid and Lipofectamine ${ }^{\mathrm{Ta}} 2000$ reagents (Life Technology, France). Selection was realized by adding $500 \mu \mathrm{g} / \mathrm{ml}$ of Geneticin selective antibiotic in complete medium of cell culture for 3 weeks. Next, 1 week was used to amplify the cells. Veracity of transfection was determined by PCR analyses using primers directed against pcDNA3.3 (ACGTTGTCACTGAAGCGG and CCTGATGCTCTTCGTCCA) and by the fact that each plasmid affects the DNMT1/protein-x interaction of interest.

\section{Measure of the 5-methylcytosine level}

DNA was extracted using the QiaAmp DNA mini Kit (Qiagen, France). The quantification of 5-methylcytosine is performed by using the Methylamp Global DNA methylation Quantification kit (Euromedex-Epigenetiek, France).

\section{Tumorigenicity assay}

Cultured cells were harvested by trypsinization, washed and resuspended in saline buffer. Cell suspensions were injected s.c. as $10^{6}$ cells in $0.2 \mathrm{ml}$ volume in the flank of 7-/8-week-old nude NMRI-nu female mice (Janvier, France).

\section{Additional files}

Additional file 1: Figure S1. Representation of the pcDNA3.3 plasmid used to express the indicated peptides in cells. For each peptide, the amino acid position, sequences and corresponding cDNA sequences are indicated in the table.

Additional file 2: Figure S2. Graphs illustrate the impact of the expression of the considered peptide on the indicated DNMT1/protein-x interaction of close proximity. Graph illustrates the average \pm SD obtained from 100 cells in three independent experiments. ${ }^{*}$ Only the corresponding interaction was significantly decreased ( $p<0.05, t$-test) by the considered peptides in comparison with the data obtained from untreated cells. "Untreated" indicates that cells are not transfected by a plasmid encoding for a peptide or are not treated with a DNMT inhibitor.
Thus, this condition is used as a control. For each peptide, the specificity of inhibition of disruption of one considered peptide is reinforced by the use of six other peptides.

Additional file 3: Figure S3. Schematic representation of the $\mathrm{TMZ}+$ irradiation treatment administered to the cells.

Additional file 4: Figure S4. Schematic representation of the treatments administered to mice.

Additional file 5: Figure S5. Effect of $47-60^{\mathrm{DNMT} 1}$ and $\mathrm{m} 47-60^{\mathrm{DNMT} 1}$ on the DNMT1/DMAP1 interaction. Graphs illustrate the impact of the considered peptide on the indicated DNMT1/protein-x interaction of close proximity. Graph illustrates the average \pm SD obtained from 100 cells in three independent experiments. ${ }^{*}$ Only the corresponding interaction was significantly decreased ( $p<0.05$, $t$-test) by the considered peptides in comparison with the data obtained from untreated cells.

\section{Competing interests}

The authors declare that they have no financial relationship with the organization that sponsored the research.

\section{Authors' contributions}

PFC initiated and conceived the study, undertook and interpreted analyses, and drafted the manuscript. FMV provided data interpretation and manuscript review. PFC, MC, RP and AN performed epigenetic laboratory studies. All authors read and approved the final manuscript.

\section{Acknowledgements}

This work was supported by grants from the Ligue contre le cancer GrandOuest des comités départementaux de Loire-Atlantique, de Vendée, lle et Vilaine et du Maine et Loire (Subvention 2011 et Subvention 2013) and from the Canceropôle Grand Ouest.

\section{Author details}

'Centre de Recherche en Cancérologie Nantes-Angers, INSERM, U892, Equipe Apoptose et progression tumorale, Equipe labellisée Ligue Nationale Contre le Cancer, 8 quai moncousu, BP7021, 44007, Nantes, France. ²Département de Recherche en Cancérologie, Université de Nantes, Faculté de Médecine, IFR26, F-4400, Nantes, France. ' ${ }^{2} \mathrm{LaBCT}$, Institut de Cancérologie de l'Ouest, Boulevard J Monod, 44805, Nantes, Saint Herblain Cedex, France. ${ }^{4}$ Institut de Recherche Thérapeutique INSERM U892 - CRCNA, Equipe 9 -Apoptose et Progression tumorale, 8 Quai Moncousu, BP 70721, 44007, Nantes, Cedex 1, France.

Received: 19 March 2013 Accepted: 3 June 2013

Published: 28 June 2013

\section{References}

1. Eden A, Gaudet F, Waghmare A, Jaenisch R: Chromosomal instability and tumors promoted by DNA hypomethylation. Science 2003, 300(5618):455.

2. Gaudet F, Hodgson JG, Eden A, Jackson-Grusby L, Dausman J, et al: Induction of tumors in mice by genomic hypomethylation. Science 2003, 300(5618):489-492.

3. Hervouet E, Lalier L, Debien E, Cheray M, Geairon A, et al: Tumor induction by disruption of the Dnmt1, PCNA and UHRF1 interactions. Nature Precedings 2008. http://hdl.handle.net/10101/npre.2008.2509.1.

4. Hervouet $E$, Debien E, Cheray M, Hulin P, Loussouarn D, et al: Disruption of Dnmt1/PCNA/UHRF1 interactions promotes tumorigenesis by inducing genome and gene-specific hypomethylations and chromosomal instability. PLoS One 2010, 5(6):e11333.

5. Bostick M, Kim J, Estève P, Clark A, Pradhan $S$, et al: UHRF1 plays a role in maintaining DNA methylation in mammalian cells. Science 2007, 27(15): 2187-2197.

6. Estève $\mathrm{P}$, Chin $\mathrm{H}$, Pradhan $\mathrm{S}$ : Human maintenance DNA (cytosine-5)methyltransferase and p53 modulate expression of p53-repressed promoters. Proc Natl Acad Sci U S A 2005, 102(4):1000-1005.

7. Estève $P$, Chin H, Pradhan S: Molecular mechanisms of transactivation and doxorubicin-mediated repression of survivin gene in cancer cells. J Biol Chem 2007, 282(4):2616-2625. 
8. Fuks F, Hurd P, Deplus R, Kouzarides T: The DNA methyltransferases associate with $\mathrm{HP} 1$ and the SUV39H1 histone methyltransferase. Nucleic Acids Res 2003, 31(9):2305-2312.

9. Fuks F, Burgers WA, Brehm A, Hughes-Davies L, Kouzarides T: DNA methyltransferase Dnmt1 associates with histone deacetylase activity. Nat Genet 2000, 24(1):88-91.

10. Hervouet E, Vallette FM, Cartron PF: Dnmt1/transcription factor interactions: an alternative mechanism of DNA methylation inheritance. Genes \& Cancer 2010, 1(5):434-443.

11. Margot JB, Ehrenhofer-Murray AE, Leonhardt H: Interactions within the mammalian DNA methyltransferase family. BMC Mol Biol 2003, 4(1):7.

12. Muromoto R, Sugiyama K, Takachi A, Imoto S, Sato N, et al: Physical and functional interactions between Daxx and DNA methyltransferase 1-associated protein, DMAP1. J Immunol 2004, 172(5):2985-2993.

13. Sharif J, Muto M, Takebayashi S, Suetake I, Iwamatsu A, et al: The SRA protein Np95 mediates epigenetic inheritance by recruiting Dnmt1 to methylated DNA. Nature 2007, 450(7171):908-912.

14. Viré E, Brenner C, Deplus R, Blanchon L, Fraga M, et al: The Polycomb group protein EZH2 directly controls DNA methylation. Nature 2006, 439(7078):871-874

15. Kuck D, Singh N, Lyko F, Medina-Franco J: Novel and selective DNA methyltransferase inhibitors: docking-based virtual screening and experimental evaluation. Bioorg Med Chem 2010, 18(2):822-829.

16. Medina-Franco J, López-Vallejo F, Kuck D, Lyko F: Natural products as DNA methyltransferase inhibitors: a computer-aided discovery approach. Mol Divers 2011, 15(2):293-304.

17. Suzuki T, Tanaka R, Hamada S, Nakagawa H, Miyata N: Design, synthesis, inhibitory activity, and binding mode study of novel DNA methyltransferase 1 inhibitors. Bioorg Med Chem Lett 2010, 20(3):1124-1127.

18. Yoo J, Medina-Franco J: Inhibitors of DNA methyltransferases: insights from computational studies. Curr Med Chem 2012, 19(21):3475-3487.

19. Yoo J, Kim J, Robertson K, Medina-Franco J: Molecular modeling of inhibitors of human DNA methyltransferase with a crystal structure: discovery of a novel DNMT1 inhibitor. Adv Protein Chem Struct Biol 2012, 87:219-247

20. Issa J: Optimizing therapy with methylation inhibitors in myelodysplastic syndromes: dose, duration, and patient selection. Nat Clin Pract Oncol 2005, 2(1):S24-S29.

21. Momparler R, Côté S, Eliopoulos N: Pharmacological approach for optimization of the dose schedule of 5-Aza-2'-deoxycytidine (Decitabine) for the therapy of leukemia. Leukemia Suppl 2007, 1:S1-S6.

22. Chik F, Szyf M: Effects of specific DNMT gene depletion on cancer cell transformation and breast cancer cell invasion; toward selective DNMT inhibitors. Carcinogenesis 2010, 32(2):224-232.

23. Liu L, Ling X, Liang H, Gao Y, Yang H, et al: Hypomethylation mediated by decreased DNMTs involves in the activation of proto-oncogene MPL in TK6 cells treated with hydroquinone. Toxicol Lett 2012, 209(3):239-245.

24. Schaefer L, Schuster D, Schaffer J: Structured illumination microscopy: artefact analysis and reduction utilizing a parameter optimization approach. J Microsc 2004, 216(Pt 2):165-174.

doi:10.1186/1868-7083-5-9

Cite this article as: Cheray et al: Specific inhibition of one DNMT1including complex influences tumor initiation and progression. Clinical Epigenetics 2013 5:9.

\section{Submit your next manuscript to BioMed Central and take full advantage of:}

- Convenient online submission

- Thorough peer review

- No space constraints or color figure charges

- Immediate publication on acceptance

- Inclusion in PubMed, CAS, Scopus and Google Scholar

- Research which is freely available for redistribution 